Communication, technologies et développement

$6 \mid 2018$

Technologies mobiles, innovation et développement

\title{
Technologies mobiles : quand l'innovation vole au secours des écosystèmes
}

Mobile technologies: when innovation flies to help ecosystems

Tecnologías móviles : cuando la innovación acude en ayuda de los ecosistemas

Julien Vieira et Lise Vieira

\section{OpenEdition}

Édition électronique

URL : http://journals.openedition.org/ctd/2316

DOI : $10.4000 /$ ctd. 2316

ISSN : 2491-1437

Éditeur

Chaire Unesco Pratiques émergentes en technologies et communication pour le développement

Référence électronique

Julien Vieira et Lise Vieira, «Technologies mobiles : quand l'innovation vole au secours des

écosystèmes », Communication, technologies et développement [En ligne], 6 | 2018, mis en ligne le 18 décembre 2018, consulté le 08 septembre 2019. URL : http://journals.openedition.org/ctd/2316 ; DOI $10.4000 /$ ctd. 2316

Ce document a été généré automatiquement le 8 septembre 2019

Communication, technologies et développement 


\title{
Technologies mobiles : quand l'innovation vole au secours des écosystèmes
}

\author{
Mobile technologies: when innovation flies to help ecosystems \\ Tecnologías móviles : cuando la innovación acude en ayuda de los ecosistemas
}

\author{
Julien Vieira et Lise Vieira
}

1 En plein essor quant à leurs performances techniques les objets connectés prennent une importance croissante dans le quotidien de nos contemporains. Leurs usages se répandent de plus en plus et cela va de pair avec le développement de leurs capacités. Depuis le commencement des années 2000, les fonctions des téléphones portables se sont amplifiées. L’appareil mobile du début est devenu un véritable ordinateur de taille réduite, mais aux multiples fonctions.

2 Ces objets se diversifient, et se déclinant sous de nombreuses formes, leurs versions successives se multiplient, l'une chassant l'autre. Dans une certaine mesure ils ont une fonction d'indicateur d'appartenance sociale et sont des signes apparents de la capacité de leurs propriétaires à être dans l'air du temps (Tisseron, 1999). Bien intégrés dans les usages, certains d'entre eux acquièrent le statut d'innovation et nous accompagnent dans différentes circonstances de notre vie. C'est ainsi que la domotique a optimisé la gestion matérielle de nos habitats les plus évolués et qu'on a vu fleurir avec plus ou moins de succès les montres, lunettes et bracelets connectés. Ainsi ces objets influent sur la manière de vivre et d'agir, mais ils mettent aussi l'humain sous surveillance puisque les données collectées laissent trace.

3 Les technologies mobiles ont entraîné de notables évolutions dans de multiples champs d'application, qu'il s'agisse d'usages privés ou collectifs (Des Horts, Isaac, 2006 ; Kiyindou, 2015). Nous ne ferons qu'évoquer la nature paradoxale de l'apport des TIC à l'environnement: ce n'est que récemment que l'impact environnemental des TIC est devenu une préoccupation des gouvernements (Petit et al., 2009). Elles génèrent en effet 
des effets négatifs sur la nature comme l'émission de gaz à effet de serre (Cueugniet, 2011).

4 Cependant, ces inconvénients, bien qu'indéniables, restent à la marge. De fait, les objets connectés ont un potentiel d'innovation qui a profondément fait évoluer la protection de la nature : il est désormais possible de dresser avec grande précision l'état des lieux de la planète et de collecter des données de plus en plus nombreuses et précises. Grâce à ces avancées, des écosystèmes entiers sont ainsi mis sous surveillance (Hoekstra , 2015). Ces technologies permettent la sauvegarde des espèces végétales: les spécimens des forêts tropicales de l'Amazonie et du Congo font l'objet d'un recensement par satellite. En outre, la lutte contre les activités d'exploitation forestières clandestines est largement facilitée grâce à l'usage des smartphones.

5 La sauvegarde des espèces animales bénéficie également de ces progrès technologiques. Iain Douglas-Hamilton, fondateur de l'organisation Save the Elephants a été le premier à encourager l'utilisation des GPS et des liaisons satellitaires pour l'observation de la faune (Wittemyer, Douglas-Hamilton, 2005). Une étude sur les déplacements des éléphants a été menée dans la réserve de Lewa, au nord du Kenya, par l'usage de colliers connectés au réseau mobile Safaricom. C'est avec le satellite QuickBird qu'un recensement exhaustif de la population des manchots empereurs dans l'Antarctique a été réalisé (Fretwell, 2012). Enfin la migration des oiseaux fait aussi l'objet de multiples observations grâce à des équipements mobiles, du plus basique (le radio-pistage) au plus coûteux, la balise Argos, qui réalise la localisation quasi exacte de l'oiseau par satellite.

6 Cependant, malgré les apports indéniables qu'elles procurent à la sauvegarde de la nature, ces technologies "classiques" se trouvent désormais concurrencées par des objets volants de nouvelle génération: les drones. Selon l'INSA, le drone ou UAV ( Unmanned Aerial Vehicle) peut être défini comme

« un aéronef inhabité, piloté à distance, semi-autonome ou autonome, susceptible d'emporter différentes charges utiles le rendant capable d'effectuer des tâches spécifiques pendant une durée de vol pouvant varier en fonction de ses capacités $»^{1}$.

7 Cet engin volant se révèle l'outil idéal pour assurer une mission de surveillance du territoire. Bien que les drones aériens soient les plus connus, il existe également des drones marins et terrestres pouvant effectuer des missions de précision (Captiven, 2013).

8 L'essor spectaculaire de cette technologie mobile nous amène à nous interroger plus spécifiquement sur les enjeux révélés par son utilisation dans la protection de l'environnement. Plus précisément, il apparaît essentiel d'étudier, tant du point de vue des Sciences de l'information et de la communication que de la Science juridique, l'impact de l'usage du drone à des fins civiles sur les normes et pratiques de sauvegarde du patrimoine environnemental.

9 Cette problématique revêt un intérêt fondamental, car elle permet d'explorer plusieurs pistes sur les impacts humains et les implications juridiques de l'utilisation des technologies mobiles les plus avancées en matière d'observation et d'étude des milieux naturels sensibles. Elle amène à analyser le renouvellement des rapports de l'humain à l'espace et au temps dans la mise en œuvre de son obligation de protection de l'environnement et de maîtrise de l'urbanisme. Nous avons choisi de développer notre analyse à travers plusieurs exemples sous l'angle des enjeux écologique, fonctionnel et démocratique que révèle l'usage des drones au service des écosystèmes. 


\section{L'enjeu écologique : l'usage du drone au service de la responsabilisation de l'humain}

10 Face à la multiplication des défis écologiques, Hans Jonas (2008: 40) et Ulrick Beck (2008) ont développé la notion de responsabilité environnementale de l'humain. Cette responsabilité morale, concernant tout individu, envers les générations actuelles et futures postulant que

11 "l'existence de l'homme ne doit pas être mise en jeu » est progressivement prise en compte par le droit (Delaunay, 2012)2. La raison principale de ce phénomène de responsabilisation repose sur la difficulté technique qu'éprouvent les gouvernants face à la complexité de l'environnement qui se caractérise par l'interrelation entre l'ensemble des éléments qui composent les écosystèmes, par leur processualité ou encore par la classification des espèces (Vieira, $2017: 281$ ).

12 Les TIC permettent à l'humain d'honorer un peu plus cette responsabilité en ce qu'elles lui permettent de mieux analyser et comprendre les écosystèmes et donc de les protéger. Dans cette perspective, les usages du drone sont multiples et décuplent les potentialités de protection du patrimoine environnemental. L'encadrement juridique progressif de son utilisation à des fins civiles favorise la poursuite de cet objectif.

\section{Drones et droit : un levier pour la protection environnementale}

Depuis l'élaboration de l'arrêté du 11 avril 2012, l'utilisation des drones à des fins civiles est encadrée dans des conditions strictement définies ${ }^{3}$. Sur ce point, le droit français fait figure de précurseur dans la mesure où il a été l'un des premiers à adopter une réglementation sur l'usage des drones civils et le premier à autoriser le vol « hors de vue du pilote $»^{4}$.

14 Faisant l'objet d'une industrialisation croissante depuis 2014, le drone étend son utilité au- delà du monde militaire ${ }^{5}$. Parfois plus efficace qu'un satellite, l'UAV offre une grande diversité de possibilités pour obtenir des informations utiles à la sauvegarde de l'environnement pour différencier des espèces végétales, identifier l'habitat d'espèces animales terrestres ou aquatiques, localiser les feux de forêt ou détecter la pollution. Devenu l'outil de prédilection des géomètres, des géographes et biologistes, cet engin peut réaliser des relevés topographiques, cartographier les territoires les plus difficiles d'accès et effectuer une étude de la composition des sols (Dempuré, 2015). Par l'autorisation de l'utilisation des drones à des fins civiles, le droit a donc permis de diversifier un peu plus les modes de collecte des données géographiques et d'obtenir de ces dernières une plus grande qualité d'information.

15 On imagine dès lors ce que les fonctionnalités de cet outil peuvent apporter à l'élaboration des textes règlementaires protecteurs de l'environnement. La rédaction de documents règlementaires comme le schéma régional de cohérence écologique (SRCE) dont le but est la sauvegarde des trames vertes et bleues (TVB) ${ }^{6}$ ou les servitudes d'utilité publique comme les plans de prévention des risques naturels prévisibles (PPRNP) ${ }^{7}$ ou enfin tout document d'urbanisme, nécessite la collecte de données environnementales précises que les drones peuvent aisément fournir. 


\section{«Drone d'oiseau ${ }^{8}$}

Grâce à leur légèreté, à leur maniabilité et à leur capacité de vol à basse altitude, les drones permettent de trouver de nouveaux angles de vue augmentant notablement la qualité d'observation de l'habitat d'espèces animales. En Camargue, les chercheurs du Centre d'écologie fonctionnelle et évolutive de Montpellier utilisent les drones pour observer les colonies de flamants roses et de chevaliers aboyeurs. Une étude (Vas et al., 2015) a évalué les effets éventuels des drones sur certaines populations d'oiseaux. Après plus de plus de 200 vols au dessus des étangs de Camargue, l'analyse montre qu'il est possible de faire approcher l'engin jusqu'à $4 \mathrm{~m}$ des flamants sans effet de stress, ce qui permet d'effectuer des observations de proximité de grande précision dans une zone marécageuse difficile d'accès. vérifier si leurs populations ne sont pas en danger sans leur faire subir de perturbations. Cela marque une première étape vers un code de meilleures pratiques dans l'utilisation des drones pour la recherche écologique qui appelle à des évaluations plus approfondies et détaillées de l'impact sur la faune de ces nouvelles technologies qui permettent une optimisation de l'observation scientifique " par les possibilités de simulation et de calcul qu'elles permettent pour préserver l'environnement, par leur contribution à l'efficacité technologique et enfin comme réseau de mobilisation pour la prise de conscience et l'action écologique » (Rodhain \& Fallery, 2010).

\section{L'enjeu fonctionnel : l'usage du drone, facteur d'ubiquité de l'Homo connectus}

18 L'ubiquité est une propriété remarquable des technologies mobiles dont les drones sont une des réalisations les plus innovantes. Aujourd'hui, être un Homo connectus signifie pouvoir agir en tout lieu et à tout moment, qualité particulièrement pertinente dans le domaine des sciences environnementales où la nécessité de l'observation et de la surveillance en continu est permanente.

19 À l'origine, l'ubiquité ${ }^{9}$ est l'apanage des dieux, le don d'être présent simultanément en plusieurs lieux ${ }^{10}$. Le désir d'omniprésence correspond à un des plus anciens rêves de l'humanité : la volonté mythique de tendre vers l'absolu. Alors que pour Barthes «le mythe est une parole» (Barthes, 1970: 193), comme le précise Jacques Perriault, «la production imaginaire ne se cantonne pas dans les discours, elle conduit à des réalisations » (Perriault, 1989 : 88 ; Carré, Craipeau, 1996)

C'est Marc Weiser qui dans son article fondateur The computer for the 21st century (Weiser, 1991: 94-104) a introduit le concept d'« ubiquitous computing». Il propose de "prendre en compte l'environnement naturel des hommes" pour concevoir l'informatique "ubiquitaire ", décrite comme une troisième ère de l'informatique, après celle de l'ordinateur central et celle de l'ordinateur personnel.

21 Les sociétés contemporaines sont fortement imprégnées par les technologies numériques dont les qualités propres contribuent à faire avancer l'homme vers la réalisation de ses imaginaires. Les chercheurs en SIC se sont penchés sur les questions afférentes à la mobilité potentialisée par le numérique (Moeglin, 1996). Les équipements informatiques,

Communication, technologies et développement, 6 | 2018 
les réseaux favorisent l'ubiquité «la vraie promesse de l'internet, c'est de pouvoir être en plusieurs endroits simultanément, de vivre plusieurs vies en parallèle » (Breton, 2000 : 56)

Ainsi, l'imaginaire peut conduire à l'innovation et à la « fabrique du futur » (Musso \& al. 2007) Dans cette nouvelle configuration, l'utilisateur dispose d'appareils interconnectés, qui s'intégrent de manière "transparente", donc quasi invisible, à sa vie quotidienne. Cette vision de Weiser que l'on pouvait qualifier de futuriste il y a près de 30 ans se trouve aujourd'hui réalisée par l'intermédiaire des multiples machines «intelligentes", ces Things that think ${ }^{11}$ qui scrutent nos faits et gestes, nos habitudes et anticipent les demandes. Si cette forme d'ubiquité présente des avantages, elle peut aussi revêtir l'aspect d'une véritable intrusion, qui, si elle se double d'une évolution vers une intelligence des objets connectés peut aboutir à une perte de contrôle, voire à une dépendance de l'humain aux technologies.

Le domaine de l'aménagement et du développement durables met particulièrement en exergue la question de l'ubiquité par la sophistication des outils numériques. La mise en œuvre de l'objectif de la ville durable ou plus généralement de l'objectif de protection d'un écosystème se construit sur des temps longs alors que le développement de la vie sur un territoire se caractérise souvent par une succession de temps courts. Or pour comprendre les écosystèmes il faut inévitablement trouver le moyen de tenir compte de ces périodes éphémères. Dans ce contexte, les TIC permettent à l'humain de prendre conscience de la richesse des informations dont il dispose sur l'évolution d'un territoire (Vieira, 2014). Outre le rapport au temps, les TIC permettent également de dépasser les contraintes spatiales en ce qu'elles permettent un changement d'échelle avec la couverture de territoires plus vastes. La complexité propre à chaque écosystème découle souvent d'une transterritorialité, d'une transversalité, d'une désynchronisation des causes et des impacts ainsi que de nombreuses incertitudes scientifiques et techniques (Salles, 2009) que les TIC permettent de mieux comprendre.

\section{L'enjeu démocratique : l'usage du drone, média d'information écocitoyenne}

Parmi les différentes possibilités qu'il présente, le drone est devenu un moyen primordial d'obtenir des données géographiques. Dans ce domaine, il est indéniable que le droit français, via l'arrêté du 11 avril 2012, a permis de mettre à profit cette nouvelle révolution technologique qui, par extension, présente de grandes opportunités de mettre en œuvre la démocratisation informationnelle.

Cette dimension démocratique est particulièrement présente dans la définition de l'impératif de gestion des risques environnementaux. Ainsi, Michel Callon, Pierre Lascoumes et Yannick Barthes donnent au citoyen une place centrale dans la gestion de l'incertain par le recours aux procédures participatives tout en bravant les distinctions classiquement opérées entre experts et profanes (Callon et al. 2014: 380-384). L'incertitude qui plane autour de l'irréversibilité de phénomènes implique une prise de décision collective pour fixer des limites à l'activité humaine (Azam, 2006). C'est la raison pour laquelle, le droit français a notamment à travers l'article 7 de la Charte de l'environnement consacré les dispositions selon lesquelles «toute personne a le droit, dans les conditions et les limites définies par la loi, d'accéder aux informations relatives à 
l'environnement détenues par les autorités publiques et de participer à l'élaboration des décisions publiques ayant une incidence sur l'environnement ».

Progressivement, les gouvernants ont recours à l'utilisation du drone dans le cadre de la protection des écosystèmes ce qui leur permet d'assurer une information environnementale des citoyens plus précise. L'exemple de l'usage des drones pour la prévention des risques sur le littoral aquitain montre que les données collectées à l'aide de ces engins sont primordiales tant pour la sécurité environnementale que pour la mise en œuvre de l'écocitoyenneté. S'étirant sur près de $700 \mathrm{~km}$, la côte aquitaine est particulièrement touchée par l'érosion. La dune côtière qui subit les assauts de l'océan est fragilisée ce qui crée de réels dangers dans les zones habitées.

C'est pourquoi le Conseil Régional d'Aquitaine a décidé de cofinancer EarthLab Aquitaine, premier centre européen de géo information appliquée à l'environnement. Inauguré en 2013, EarthLab exerce une activité de télédétection et exploite des données collectées par satellite et drone. Privilégié pour sa précision, le drone sert à capter les détails morphologiques du terrain. Il s'agit d'identifier les indicateurs géomorphologiques du trait de côte afin de suivre son évolution pour quantifier l'importance de l'érosion et analyser les zones à risques. Une fois collectées, les données peuvent être modélisées et l'environnement de la plage est reproduit en 3 dimensions.

L'ensemble de ces données et informations est ensuite transmis aux collectivités territoriales afin de prendre des mesures face à l'érosion de la côte, le but étant d'adapter les stratégies d'aménagement : de la défense du territoire via une digue à la relocalisation des habitats en cas de danger. Ces données géographiques ont donc une fonction informative tant pour les gouvernants que pour les citoyens. Elles peuvent être utilisées dans le cadre de l'élaboration des documents d'urbanisme soumis à concertation du public: elles servent à la réalisation des documents graphiques des PLU (Plan Local d'Urbanisme) ou encore des PPRNP. Enfin, ces données sont communicables aux citoyens par un service d'open data. Mais on est encore au début du processus : les modalités de diffusion de ces données au grand public et de leur vulgarisation pour une lisibilité par le plus grand nombre restent encore à définir par le droit. L'usage du drone pour la collecte des données environnementales présente donc un réel enjeu dans la prise en compte des impératifs d'une démocratie durable et il n'est pas dit que, dans quelques années, le droit ne s'en saisisse pas dans ce domaine précis.

\section{Perspectives}

Le marché des drones dans le monde est aujourd'hui en pleine expansion. La marge de progression de ces appareils est très importante, en particulier pour la surveillance environnementale. Grâce aux protocoles soigneusement établis, les scientifiques exploiteront dans un futur proche les nombreuses possibilités qu'ils offrent, ouvrant ainsi la perspective de nouvelles applications.

Cependant, il convient de rester vigilant quant à l'utilisation de ces appareils qui portent en eux les défauts de leurs qualités : eu égard à leur extrême mobilité et à leurs capacités techniques d'approche et de précision, leurs fonctions peuvent être facilement détournées. Outils innovants de surveillance et de protection en particulier dans le domaine environnemental, ils peuvent devenir de redoutables engins-espions, s'ils tombent aux mains de personnes malintentionnées. 
qu'elles fassent progressivement l'objet de consécrations tant dans le domaine administratif, civil que pénal, un approfondissement des normes reste à accomplir pour que les usages puissent s'établir dans les meilleures conditions possibles dans le respect des libertés fondamentales.

Florence Rodhain, Bernard Fallery, Après la prise de conscience écologique, les TIC en quête de responsabilité sociale, in 15e Congrès de l'AIM, 2010 https://hal.archives-ouvertes.fr/ hal-00821450/document

\section{BIBLIOGRAPHIE}

\section{Bibliographie}

Geneviève Azam, « Développement durable-Principe de précaution ? Un nécessaire changement de paradigme ", in E. Berr et J.-M. Harribey (dir.), Le développement en question(s), Pessac, Presses universitaires de Bordeaux, coll. « Économie », 2006, p 191- 207.

Ulrich Beck, La société du risque : sur la voie d'une autre modernité, Flammarion, 2008.

Philippe Breton, Le culte de l'Internet : une menace pour le lien social ? La Découverte, 2000.

Michel Callon, Pierre Lascoumes, Yannick Barthe, Agir dans un monde incertain : essai sur ladémocratie technique, Points, 2014.

Captiven, Les drones pour la surveillance environnementale, 2013

http://www.captiven.fr/sites/default/files/pictures/Note\%20de\%

20veille_Drones_Surveillance_Environnement \%20- \%20Tech2Market_V3IC_VFok.pdf

Dominique Carré, Sylvie Craipeau, « Entre délocalisation et mobilité : analyse des stratégies entrepreneuriales de télétravail », Technologies de l'Information et Société, 1996, vol. 8, no 4, p 333-354.

Jean Cueugniet, « La contribution des éco-TIC à la protection de l'environnement », Annales des Mines-Responsabilité et environnement, No 1, ESKA, 2011, p 64-70

http://www.cairn.info/revue-responsabilite-et-environnement1-2011-1-page-64.html\#no2 consulté le 11 mai 2017.

Bénédicte Delaunay, «Les fondements d'une responsabilité (I) - Le fait générateur de la responsabilité envers les générations futures », in J.-P. Markus (dir.), Quelle responsabilité juridique envers les générations futures?, Dalloz, coll. « Thèmes \& commentaires », 2012, p 213-225.

Frédéric Dempuré, « Bienvenue dans le monde des drones », La Semaine Juridique Notariale et Immobilière, no 31-35, Juillet 2015.

Peter T. Fretwell, Michelle A.Larue, Paul Morin et al. « An emperor penguin population estimate : the first global, synoptic survey of a species from space. » PLoS One, 2012, vol. 7, no 4. 
Jon Hoekstra, La nature connectée : technologie et protection de l'environnement, 2015, http:// future.arte.tv/fr/la-nature-connectee-comment-la-technologie-transforme-la-protection-delenvironnement consulté le 11 mai 2017.

Charles-Henri Besseyre Des Horts, Henri Isaac, «L'impact des TIC mobiles sur les activités des professionnels en entreprise », Revue française de gestion, 2006, no 9, p. 243-263.

Patrice Flichy, «L'individualisme connecté entre la technique numérique et la société ", Réseaux, 2004, no 2, p 17-51.

Hans Jonas, Le principe responsabilité : une éthique pour la civilisation technologique, Flammarion, 2008.

Alain Kiyindou, Lancement de la chaire Unesco Pratiques émergentes des technologies et communication pour le développement-Université Bordeaux-Montaigne, Revue française des sciences de l'information et de la communication, (6). 2015.

Bruno Latour, Politiques de la nature : comment faire entrer les sciences en démocratie, La Découverte, 2016.

Pierre Moeglin, La mobilité entre ubiquité et omniprésence, Actes des séminaires Actions scientifiques, 1996 , no 6.

Thomas More, Utopia (1516), Scolar Press Ltd., 1966.

Pierre Musso, Laurent Ponthou, Eric Seulliet, Fabriquer le futur 2 : L'imaginaire au service de l'innovation, Pearson Education France, 2007.

Jacques Perriault, La logique de l'usage. Essai sur les machines à communiquer, Flammarion, 1989.

Michel Petit, Henri Breuil, Jean Cueugniet, Développement Eco-responsable et TIC (DETIC), Rapport du Conseil Général de l'Industrie, de l'Énergie et des Technologies, La Documentation Française, 2009.

Edouard Pflimlin, Drones civils, le décollage, 2013 http://www.mondediplomatique.fr/2013/12/ pflimlin/49974

Denis Salles, Environnement : la gouvernance par la responsabilité ?, Vertigo, 2009, hors-série 6.

Serge Tisseron, Comment l'esprit vient aux objets, Aubier, 1999. Elisabeth Vas, Amélie Lescroël, Olivier Duriez, Guillaume Boguszewski,

David Grémillet, « Approaching birds with drones : first experiments and ethical guidelines », Biology letters, 11(2), 2015 http://rsbl.royalsocietypublishing.org/content/11/2/20140754.

Julien Vieira, Écocitoyenneté et démocratie environnementale, Thèse de droit, Université de Bordeaux, 2017.

Julien Vieira, « Révolution numérique et droit de l'homme à un environnement sain », in Pauline Milon et David Samson (dir.), Révolution juridique, révolution scientifique : vers une fondamentalisation $d u$ droit de l'environnement? Actes du colloque tenu le 25 janvier 2013 à l'Université d'AixMarseille, Presses universitaires d'Aix-Marseille, 2014, p 177-204.

Marc Weiser, The computer for the 21st century, Scientific american, 265(3), 1991, p 94- 104.

George Wittemyer, Iain Douglas-hamilton, W. M Getz, « The socioecology of elephants : analysis of the processes creating multitiered social structures ", Animal behaviour, 2005, vol. 69, no 6, p 1357-1371. 


\section{NOTES}

1. Institut national des sciences appliquées https://www.insa-strasbourg.fr/fr/le-drone consulté le 7 mai 2017.

2. L'article 2 de la Charte de l'environnement faisant partie de la Constitution française de 1958 dispose que «toute personne a le devoir de prendre part à la préservation et à l'amé- lioration de l'environnement».

3. Arrêté du 11 avril 2012 relatif à l'utilisation de l'espace aérien par les aéronefs qui circulent sans personne à bord, JORF $n^{\circ} 0109$ du 10 mai 2012, p. 8655, texte $n^{\circ} 9$. Il a été abrogé par les arrêtés du 17 décembre 2015 : l'arrêté relatif à la conception des aéronefs civils qui circulent sans personne à bord, aux conditions de leur emploi et aux capacités requises des personnes qui les utilisent, JORF $n^{\circ} 0298$ du 24 décembre 2015 p. 23897, texte $n^{\circ} 22$ et l'arrêté relatif à l'utilisation de l'espace aérien par les aéronefs qui circulent sans personne à bord, JORF $\mathrm{n}^{\circ} 0298$ du 24 décembre 2015 p. 23890 , texte $n^{\circ} 20$. L'élaboration de ces deux textes a spécifiquement pour conséquence une extension du périmètre de certains des scénarios opérationnels prédéfinis ainsi qu'une simplification des démarches de demande d'autorisation de vol.

4. 2014, année des mini-drones?, http://www.leparisien.fr/high-tech/2014-annee-des-minidrones-29-12-2013-3447469.php consulté le 7 mai 2017

5. Seulement $10 \%$ des drones sont à usage civil, les autres étant à vocation militaire. (Captiven, 2013).

6. Instituée par les lois «Grenelle I» et «Grenelle II ( 2009 et 2010), la TVB est un outil d'aménagement du territoire visant à (re) constituer des réseaux écologiques cohérents.

7. La loi Barnier du 2 février 1995 relative au renforcement de la protection de l'environ- nement a mis en place les PPRNP appelés à délimiter des zones exposées aux risques et à règlementer les constructions, travaux instal- lations, aménagements ainsi que les mesures de prévention, de protection et de sauvegarde. Les plans de prévention des risques d'inon- dations (PPRI) ou les plans d'incendies de forêt (PPRIF) sont deux exemples courants de PPRNP.

8. Nous empruntons ce titre à un article Publié par LUM Le magazine science et société de l'Université de Montpellier, le 26 mai 2016. https://www.echosciences-sud.fr/articles/drone-doiseau consulté le 11 mai 2017.

9. Alors que le nomadisme permet à l'usager de se connecter dans ses déplace- ments depuis différents lieux sans que la connexion soit permanente, l'ubiquité lui donne cette dimension d'omniprésence. Notons aussi le terme de «mobiquité » inventé par Xavier Dalloz, consultant en utilisation stratégique des TIC, qui est à l'origine du concept ATAWADAC (AnyTime, AnyWhere, AnyDevice, Any Content).

10. Alors qu'on pourrait la considérer comme une forme d'utopie, qui signifie « nulle part » selon le terme créé par Thomas More en 1516, l'ubiquité désigne au contraire le " partout » 11. Consortium de MIT Media Lab qui analyse les fonctionnalités des objets augmentés. (Musso, $2007: 113)$ 


\section{RÉSUMÉS}

L'ubiquité est une propriété remarquable des technologies mobiles qui initient de nouvelles pratiques dans le domaine de la protection de la nature. Fondés sur le numérique, les objets connectés changent indéniablement la donne en dotant les défenseurs de la nature de capacités accrues de surveillance et d'analyse. Cela permet ainsi de suivre le rythme de l'un des défis les plus importants de notre ère: la sauvegarde du patrimoine environnemental.Parmi ces technologies, les drones sont une des réalisations les plus innovantes. Notre propos est de poser la question de l'impact de leur usage à des fins civiles sur les normes et pratiques de sauvegarde $\mathrm{du}$ patrimoine environnemental. Quels en sont les impacts, les limites, et quelles perspectives peut- on voir se dessiner? Cette analyse sur les implications de l'utilisation de ces technologies mobiles est effectuée dans une double approche informationnelle et juridique.

Ubiquity is a remarkable property of mobile technologies that initiate new practices in the field of nature protection. Digitally based, connected objects undeniably change the situation by giving the defenders of nature better monitoring and analysis capabilities. This helps to keep pace with one of the most important challenges of our era: safeguarding environmental heritage. Among these technologies, drones are one of the most innovative achievements. Our purpose is to ask the question of the impact of their use for civil purposes on standards and practices for safeguarding the environmental heritage. What are the impacts, the limits, and what perspectives can we foresee? This analysis of the implications of the use of these mobile technologies is carried out in a dual informational and legal approach.

La ubicuidad es una propiedad notable de las tecnologías móviles que inician nuevas prácticas en el campo de la protección de la naturaleza. Los objetos conectados basados en digital están cambiando la situación dando a los defensores de la naturaleza más recursos de monitoreo y análisis. Esto ayuda a mantenerse al día con uno de los desafíos más importantes de nuestra era : salvaguardar el patrimonio ambiental.

Entreestastecnologías, losdronesson uno de los logros más innovadores. Nuestro propósito es plantear la cuestión del impacto de su uso para fines civiles en las normas y prácticas para salvaguardar el patrimonio ambiental. ¿Cuáles son los impactos, los límites y las perspectivas que podemos ver ? Este análisis de las implicaciones del uso de estas tecnologías móviles se realiza en un doble enfoque informativo y legal.

\section{INDEX}

Mots-clés : technologies mobiles, drones, protection, environnement, ubiquité

Keywords : mobile technologies, drones, protection, environment, ubiquity

Palabras claves : tecnologías móviles, drones, proteccion, medio ambiente, ubicuidad 


\section{AUTEURS}

\section{JULIEN VIEIRA}

Centre Léon Duguit, Université de Bordeaux

\section{LISE VIEIRA}

MICA-Université Bordeaux- Montaigne 